\title{
Increasing the Quality and Availability of Evidence-based Treatment for Tobacco Dependence through Unified Certification of Tobacco Treatment Specialists
}

\author{
Christine E. Sheffer, ${ }^{1}$ Thomas Payne, ${ }^{2}$ Jamie S. Ostroff, ${ }^{3}$ Denise Jolicoeur, ${ }^{4}$ Michael Steinberg, ${ }^{5}$ \\ Sharon Czabafy, ${ }^{6}$ Jonathan Foulds, ${ }^{7}$ Matthew Bars, ${ }^{8}$ Ken Wassum, ${ }^{9}$ and Barbara Perry ${ }^{10}$ \\ (Training Issues Committee of the Association for the Treatment of Tobacco Use and \\ Dependence) \\ ${ }^{1}$ Community Health and Social Medicine Department, Sophie Davis School of Biomedical Education, City College of New York, \\ Harris Hall Suite 400, 160 Convent Avenue, New York, NY \\ 2 Department of Otolaryngology and Communicative Sciences, Jackson Medical Mall Suite 611, University of Mississippi Medical \\ Center, 350 West Woodrow Wilson Avenue, Jackson, Mississippi \\ ${ }^{3}$ Department of Psychiatry \& Behavioral Sciences, Memorial Sloan-Kettering Cancer Center, 641 Lexington Avenue, New York, \\ NY (Center for Tobacco Treatment Research and Training) \\ ${ }^{4}$ Preventive and Behavioral Medicine, University of Massachusetts Medical School 55 Lake Avenue North, Worcester, \\ Massachusetts \\ ${ }^{5}$ Division of General Internal Medicine, Rutgers-Robert Wood Johnson Medical School, 125 Paterson St, Suite 2300, New \\ Brunswick, New Jersey \\ ${ }^{6}$ Ephrata Community Hospital Wellness Center, 63 West Church Street, Stevens, Pennsylvania \\ ${ }^{7}$ College of Medicine, Cancer Institute, Penn State University, Cancer Control Program, Hershey, Pennsylvania \\ ${ }^{8}$ Fire Department of the City of New York Tobacco Treatment Program, World Trade Center Medical Monitoring \& Treatment \\ Program 9 Metrotech Center, Brooklyn, New York \\ ${ }^{9}$ Alere, 999 Third Avenue Suite 2100, Seattle, WA \\ ${ }^{10}$ MMC Tobacco Treatment Program \& MaineHealth Center for Tobacco Independence, 110 Free Street Portland, Maine
}

Each year, tobacco use causes over 6 million deaths and is responsible for hundreds of billions of dollars in health care and economic costs in the world (WHO, 2011). If current trends continue, tobacco is expected to kill over 1 billion people in the $21^{\text {st }}$ century, making it one of the single greatest causes of preventable death and disease in history (WHO, 2011). Long-term abstinence from tobacco use dramatically improves individuals' health, reduces the incidence of tobacco-related disease, and is clearly responsible for saving lives (Anthonisen et al., 2005). Most tobacco users express a desire to achieve long-term abstinence from tobacco use and make numerous unsuccessful quit attempts over the course of many years (Borland, Partos, Yong, Cummings, \& Hyland, 2012; CDC, 2011). Evidence-based treatments for tobacco use and dependence greatly improve the chances that quit attempts result in long-term abstinence (Chambless \& Hollon, 1998; Chambless et al., 1998; Compas, Haaga, Keefe, Leitenberg,
\& Williams, 1998; Fiore et al., 2008; Zwar et al., 2004). Increasing the availability of high-quality evidence-based treatment for tobacco use and dependence will make it more likely that tobacco users use evidence-based treatments and that quit attempts translate into long-term abstinence. The professionalisation of treatment for tobacco dependence by the development of a rigorous, unified Tobacco Treatment Specialist (TTS) certification process will increase the availability of high-quality evidence-based treatment for tobacco use and dependence for all tobacco users.

Most tobacco users express a desire to achieve longterm abstinence from tobacco; over $90 \%$ of smokers make regular, albeit unsuccessful, quit attempts over the course of many years of smoking (Borland et al., 2012; CDC, 2011), but overall only about $4.3 \%$ of smokers are able to maintain abstinence 6-12 months after an unassisted quit attempt (Hughes et al., 1992; Levy, Graham, Mabry, 
Abrams, \& Orleans, 2010). Smokers who use evidencebased behavioural counselling and/or medications are over three times more likely to achieve long-term abstinence than those who do not (Anthonisen et al., 2005; Fiore et al., 2008; Kasza et al., 2013). Nonetheless, in the United States and elsewhere, few tobacco users use evidence-based treatments when they attempt to quit (CDC, 2011). In the United States for example, less than one third of smokers use any form of evidence-based treatment and less than $5 \%$ use the most effective treatment, evidence-based medication and counselling (CDC, 2011). Given the opportunities provided by a significant reach into the smoking population, the clinical practice guidelines in many countries strongly recommend that health care practitioners briefly discuss evidence-based treatment options with all tobacco users at every visit (CAN-ADAPTT, 2011; Fiore et al., 2008; Health, 2007; NICE, 2013; ). Practitioners, however, are generally unprepared to effectively discuss treatment options and are unfamiliar with the high intensity of treatment provided by TTSs (Applegate, Sheffer, Crews, Payne, \& Smith, 2008; Payne et al., 2014; Sheffer, Anders, Brackman, Steinberg, \& Barone, 2012; Steinberg, Alvarez, Delnevo, Kaufman, \& Cantor, 2006).

The Association for the Treatment of Tobacco Use and Dependence (ATTUD), policymakers, practitioners, researchers, and other key professional organisations have long advocated for increasing the availability and ensuring the quality of evidence-based treatments for tobacco dependence provided to tobacco users within the health care system (Fiore et al., 2008). Consistent with the push-pull capacity dissemination model (Orleans, 2007), we recognise that there are multiple pathways to achieving this goal and we contend that unified certification of TTSs is central to this achievement. Unified TTS certification, developed from rigorous tobacco dependence training program accreditation and independent individual certification process will: (1) recognise and further develop a work force with the appropriate level of expertise to train health care providers in low-intensity tobacco dependence treatment; (2) support health care teams in the systematic delivery of evidence-based treatment; (3) ensure that all tobacco users are provided with a comprehensive, evidence-based treatment for tobacco dependence of the appropriate intensity; (4) support greater integration of evidence-based treatment for tobacco use and dependence into health care; and (5) enhance public education resources about the availability, efficacy, and safety of evidence-based treatments for tobacco use and dependence. In turn, these components will ensure that quit attempts are more likely to result in long-term abstinence.

Delivering an effective treatment for tobacco dependence requires the development of specialised knowledge, skills, and competencies commensurate with the level of intensity of the treatment to be provided. In 2005, in a multidisciplinary effort, ATTUD established a comprehensive set of Core Competencies needed by all practitioners, re- gardless of background, to deliver evidence-based treatment of tobacco dependence at varying levels of intensity. Knowledge and skill is needed to deliver a minimal, low-intensity treatment, but competencies or abilities that extend beyond skill development are required to deliver a higher intensity treatment. See Table 1 . For example, a practitioner needs specific knowledge and skills to provide a low intensity treatment such as recommending a particular medication or providing a quitting 'tip.' Many tobacco users can be effectively treated with a lower intensity treatment, but many tobacco users need a more comprehensive, higher intensity treatment to achieve long-term abstinence. To effectively provide a high intensity, comprehensive treatment, however, a practitioner requires the appropriate analytical and interpretive abilities to understand the biopsychosocial factors affecting treatment outcomes (e.g., motivation, self-efficacy, behavioral capacity, stress and coping resources, social support, environmental influences, nicotine withdrawal, mood management, etc.); solicit and cultivate the involvement and motivation of tobacco users; and apply an appropriate multifaceted intervention. Smokers treated by practitioners with more extensive training are more likely to achieve long-term abstinence among smokers treated by practitioners without training (McDermott, Beard, Brose, West, \& McEwen, 2013). These competencies are becoming increasingly important as the demographics and clinical characteristics of tobacco using population shifts and tobacco users more frequently present with comorbid medical and psychiatric conditions and use an ever evolving variety of new, untested tobacco products, and nicotine delivery devices.

The ATTUD Core Competencies for the treatment of tobacco dependence provide a foundation for the development of a rigorous TTS credential. These Core Competencies were constructed in terms of the proficiency recommended to perform varying levels of treatment intensity and thus reflect three levels of competence in terms of the following: Awareness reflects a basic level of mastery demonstrated when practitioners are able to identify the concept or skill, but have limited ability to perform the skill. Knowledge reflects an intermediate level of mastery demonstrated when practitioners are able to apply and describe the skill. Proficiency reflects an advanced level of mastery demonstrated when practitioners are able to synthesise, critique, and teach the skill. ATTUD endorses the development of a rigorous, unified process for credentialing TTSs who are trained at the proficiency level. We envision the unified TTS credential to function similarly to the credentialing developed for other specialties such as certified diabetes educator, certified asthma educator, and certified health education specialist.

The TTS credential will indicate expert-level knowledge and skills in the evidence-based treatment of tobacco dependence and how to effectively treat a diverse population of tobacco users in multiple modalities (e.g., individual, group, telephone, internet, etc.). TTSs 


\section{Table 1}

Core Competencies for evidence-based treatment of tobacco use and dependence as recommended by the Association for the Treatment of Tobacco Use and Dependence. Level of proficiency required for Brief treatment: awareness-knowledge; Intensive treatment: knowledge-proficiency

Core Competency Skill set:

1. Tobacco Dependence Knowledge and Education: Provide clear and accurate information about tobacco use, strategies for quitting, the scope of the health impact on the population, the causes and consequences of tobacco use

2. Counselling Skills: Demonstrate effective application of counselling theories and strategies to establish a collaborative relationship, and to facilitate client involvement in treatment and commitment to change

\section{Assessment Interview: Conduct an} assessment interview to obtain comprehensive and accurate data needed for treatment planning
4. Treatment Planning: Demonstrate the ability to develop an individualised treatment plan using evidence-based treatment strategies a. Describe the prevalence and patterns of tobacco use, dependence and cessation in the country and region in which the treatment is provided, and how rates vary across demographic, economic and cultural subgroups.

b. Explain the role of treatment for tobacco use and dependence within a comprehensive tobacco control program.

c. Utilise the findings of national reports, research studies, and guidelines on tobacco treatment.

d. Explain the societal and environmental factors that promote and inhibit the spread of tobacco use and dependence.

e. Explain the health consequences of tobacco use and benefits of quitting, and the basic mechanisms of the more common tobacco induced disorders.

f. Describe how tobacco dependence develops and be able to explain the biological, psychological, and social causes of tobacco dependence.

g. Summarise and be able to apply valid and reliable diagnostic criteria for tobacco dependence.

h. Describe the chronic-relapsing nature of tobacco dependence, including typical relapse patterns, and predisposing factors.

i. Provide information that is gender, age, and culturally sensitive and appropriate to learning style and abilities.

j. Identify evidence-based treatment strategies and the pros and cons for each strategy.

k. Be able to discuss alternative therapies such as harm reduction, hypnosis and acupuncture.

I. Demonstrate ability to access information on the above topics.

a. Demonstrate effective counselling skills such as active listening and empathy that facilitate the treatment process.

b. Demonstrate establishing a warm, confidential, and non-judgmental counselling environment.

c. Describe and demonstrate use of an evidence-based method for brief interventions for treating tobacco use and dependence, as identified in current guidelines.

d. Describe the use of models of behaviour change including motivational interviewing, cognitive therapy, and supportive counselling.

e. Demonstrate the effective use of clinically sound strategies to enhance motivation and encourage commitment to change.

f. Demonstrate competence in at least one of the empirically supported counselling modalities such as individual, group and telephone counseling.

a. Demonstrate the ability to conduct an intake assessment interview including:

i. tobacco use history

ii. validated measures of motivation to quit

iii. validated measures for assessing tobacco use and dependence

iv. current challenges and barriers to attaining permanent abstinence

v. current strengths to support abstinence.

vi. prior quit attempts including treatment experiences, successes and barriers

vii. availability of social support systems

viii. preferences for treatment.

ix. cultural factors influencing making a quit attempt

b. Demonstrate the ability to gather basic medical history information and conduct a brief screening for psychiatric and substance abuse issues.

c. Describe when to consult with primary medical care providers and make appropriate referrals before treatment planning is implemented.

d. Describe the existing objective measures of tobacco use such as CO monitoring, and cotinine level assessments.

a. In collaboration with the client, identify specific and measurable treatment objectives.

b. Plan-individualised treatments that account for patient assessment factors identified during the intake assessment and history gathering.

c. Collaboratively develop a treatment plan that uses evidence-based strategies to assist the client in moving toward a quit attempt, and/or continued abstinence from tobacco.

d. Describe a plan for follow up to address potential issues including negative outcomes.

e. Demonstrate the process to make referrals to other practitioners or to recommend additional care. 


\section{Table 1}

Continued

\section{Core Competency}

5. Pharmacotherapy: Provide clear and accurate information about pharmacotherapy options available and their therapeutic use
6. Relapse Prevention: Offer methods to reduce relapse and provide ongoing support for tobacco-dependent persons

\section{Diversity and Specific Health Issues:} Demonstrate competence in working with population subgroups and those who have specific health issues

8. Documentation and Evaluation: Describe and use methods for tracking individual progress, record keeping, program documentation, outcome measurement and reporting

9. Professional Resources: Utilise resources available for client support and for professional education or consultation
Skill set:

a. Describe the benefits of combining pharmacotherapy and counselling.

b. Provide information on correct use, efficacy, adverse events, contraindications, known side effects and exclusions for all tobacco dependence medications approved by national regulatory agencies.

c. Identify information relevant to a client's current and past medical, psychiatric, and smoking history,(including past treatments) that may impact pharmacotherapy decisions.

d. Provide appropriate patient education for therapeutic choices and dosing for a wide range of patient situations.

e. Communicate the symptoms, duration, incidence and magnitude of nicotine withdrawal.

f. Describe the use of combinations of medications and higher dose medications to enhance the probability of abstinence.

g. Identify second-line medications and be able to find information about them as needed.

h. Identify possible adverse reactions and complications related to the use of pharmacotherapy for tobacco dependence, making timely referrals to medical professionals/services. Demonstrate ability to address concerns about minor and/or temporary side effects of these pharmacotherapies.

i. Demonstrate ability to collaborate with other healthcare providers to coordinate the appropriate use of medications, especially in the presence of medical or psychiatric co-morbidities.

j. Provide information about alternative therapies based upon recognized reviews of effectiveness such as the Cochrane reviews and the USPHS Guidelines.

a. Identify personal risk factors and incorporate into the treatment plan.

b. Describe strategies and coping skills that can reduce relapse risk.

c. Provide guidance in modifying the treatment plan to reduce the risk of relapse throughout the course of treatment.

d. Describe a plan for continued aftercare following initial treatment.

e. Describe how to make referrals to additional resources to reduce risk of relapse.

f. Implement treatment strategies for someone who has lapsed or relapsed.

a. Provide culturally competent counselling

b. Describe specific treatment indications for special population groups (i.e. pregnant women, adolescents, young adults, elderly, hospitalized patients, those with co-morbid psychiatric conditions). c. Demonstrate an ability to respond to high-risk client situations.

d. Make effective treatment recommendations for non-cigarette tobacco users.

e. Describe recommendations for those exposed to environmental tobacco smoke pollution.

a. Maintain accurate records utilising accepted coding practices that are appropriate to the setting where services are provided.

b. Develop and implement a protocol for tracking client follow up and progress.

c. Describe standardised methods of measuring recognised outcomes of tobacco dependence treatment for individuals and programs.

a. Describe resources (web based, community, quitlines) available for continued support for tobacco abstinence for clients.

b. Identify community resources for referral for medical, psychiatric or psychosocial problems.

c. Name and use peer-reviewed journals, professional societies, websites, and newsletters, related to tobacco dependence treatment and/or research.

d. Describe how patients can explore reimbursement for treatments.

10. Law and Ethics: Consistently use a code of ethics and adhere to government regulations specific to the health care or work site setting

11. Professional Development: Assume responsibility for continued professional development and contributing to the development of others a. Describe and use a code of ethics established by your professional discipline for tobacco dependence treatment specialists if available.

b. Describe the implications and utilise the regulations that apply to the tobacco treatment setting (confidentiality, HIPAA, work site specific regulations).

c. Maintain professional standards as required by professional license or certification.

d. Utilise the literature and other formal sources of inquiry to remain current in tobacco dependence treatment.

e. Describe the implications of current research to the practice of tobacco dependence treatment

f. Disseminate knowledge and findings about tobacco treatment with others through formal and informal channels. 
understand the importance and the role of brief, lower intensity evidence-based treatment and are professionally prepared to provide an appropriate level of training to a variety of health care providers to enable them to deliver an effective brief, lower intensity treatment. TTSs are also skilled in the integration of evidence-based treatments for tobacco dependence into health care systems and chronic care service models. We foresee an increased need for TTS certification in the United States, Canada, Australia, and many other areas of the world in the near future given the demonstrated need. In the United States alone, several provisions in the Affordable Care Act that support preventative interventions will result in a significant increase in the need for specialists who work with health care teams to effectively provide intensive, evidence-based treatment of tobacco dependence (DHHS, 2014). The unified TTS credential will denote that an individual is effectively prepared to provide and support treatment for tobacco dependence.

\section{Training Health Care Providers to Provide Brief Treatment for Tobacco Dependence}

Clinical practice guidelines recommend that all tobacco users be identified, assessed, and offered an appropriate level of care at every visit including a brief motivational intervention, a brief treatment, and/or a referral to a more intensive treatment users (CAN-ADAPTT, 2011; Fiore et al., 2008; Health, 2007; NICE, 2013). Incorporation of evidence-based treatment of tobacco dependence of varying intensities in all appropriate settings would improve the likelihood that tobacco users are offered, educated about, and use an evidence-based treatment consistent with their needs during multiple quit attempts as well as ensure that practitioners' and tobacco users' efforts have maximal impact (Fiore et al., 2008). Evidence indicates that this can be achieved through training, treatment policies, and systems' supports (Fiore et al., 2008; Levy et al., 2010). The unified TTS certification will prepare TTSs to provide training and support for health care teams and contribute to clinic and system supports for the delivery of treatment. Sufficient availability of highly proficient practitioners is necessary to train practitioners who deliver brief, low-intensity interventions. At present, the vast majority of health care practitioners have not received even the minimal training needed to effectively deliver a brief, low-intensity evidence-based treatment (Applegate et al., 2008; Sheffer et al., 2012; Steinberg et al., 2006). Sufficient availability of highly proficient TTSs is also necessary to support the efforts of health care providers who identify tobacco users in need of higher intensity treatment, to support innovative chronic care models such as the Ask-Advise-Connect (Vidrine et al., 2013), and to treat tobacco users with complex presentations who do not respond to lower intensity treatment. The availability of unified TTS certification will promote an increase in the demand for specialised tobacco dependence treatment training and the demand for specialised tobacco de- pendence treatment services and further develop a health care workforce capable of providing effective treatment for tobacco dependence at appropriately effective levels of intensity to meet the needs of an increasingly complex population of tobacco users.

\section{Public Resource}

Certified TTSs, proficient in the Core Competencies also have the capacity to serve as a resource for the public. Increasing the public's awareness and understanding of evidence-based treatment for tobacco dependence is likely to increase the degree to which tobacco users inquire about, expect to use, and purchase evidence-based medications and counselling (Orleans, 2007). This is especially important among groups who more frequently harbour misconceptions about these treatments and are less frequently offered these treatments by practitioners (Bansal, Cummings, Hyland, \& Giovino, 2004; Houston, Scarinci, Person, \& Greene, 2005; McMenamin, Halpin, \& Bellows, 2006). A number of creative educational and promotional strategies have been proposed the National Tobacco Cessation Collaborative Consumer Demand Roundtable (Backinger et al., 2010). These promotional strategies compare evidence-based treatments with non-evidencebased treatments, address tobacco users' misconceptions and concerns about evidence-based treatments, provide clear and appropriate expectations regarding the role of medications and behavioural counselling, and provide individuals with the tools for how to investigate whether or not a treatment is evidence-based (Backinger et al., 2010; Orleans, 2007; Orleans, Mabry, \& Abrams, 2010). These strategies are likely to create a more educated consumer base, increase involvement of practitioners in tobacco users' quit attempts, and increase the demand for comprehensive evidence-based treatment provided by a certified TTS (Backinger et al., 2010; Orleans et al., 2010), but this demand must be met with an adequately trained health care workforce with the appropriate resources to provide treatment of the appropriate intensity. The unified TTS credential will help achieve these objectives.

\section{Accreditation of TTS Training Programs}

In order to develop the capacity to provide the appropriate level of training for practitioners seeking a TTS credential, in 2009 ATTUD established the Council for Tobacco Treatment Training Programs (CTTTP) (www.attudaccred.org), a voluntary, multi-disciplinary entity whose goals include promoting excellence in the professional preparation of TTSs through the accreditation of TTS training programs. The CTTTP developed and now maintains a TTS training accreditation program. TTS training programs accredited by the CTTTP prepare TTSs to proficiently deliver high intensity, comprehensive, multicomponent, evidence-based treatments for tobacco dependence in a variety of contexts, and address the needs of tobacco users with complex presentations. 
The CTTTP is guided by a multidisciplinary panel of experts who review new applications and annually review all CTTTP-accredited programs. The application and review process includes demonstrating how the training experience supports the development of the Core Competencies. There are currently 12 -accredited TTS training programs in North America and several in the process of becoming accredited.

The proposed unified TTS credential is, at present, timely. TTSs are quickly becoming an established specialty (Wolff, Hughes, \& Woods, 2013); however, the processes and opportunities for individual TTS credentialing vary significantly by location. Several CTTTP-accredited training programs have sought to fill the certification void by providing a 'certificate' and/or an evaluation for trainees; however, these options do not offer the objectivity and rigour provided by an independent credentialing process and there is an inherent conflict of interest when training programs also offer credentialing. Moreover, at present, no TTS certification programs meet the criteria set forth by the National Commission for Certifying Agencies. Although two states in the United States offer TTS certification independent of a training program, the criteria for certification varies considerably and the certification is likely to lack meaning in other states. The directors of all the CTTTP-accredited training programs are uniquely aware of these issues and unanimously support the development of a unified TTS credential.

As a professional organisation whose mission is to promote increased access to evidence-based tobacco treatment of tobacco use and dependence, it should be noted that we are not suggesting that health care providers who do not have a TTS certification should be discouraged from providing evidence-based treatment. ATTUD supports unified TTS certification because it will advance and support the dissemination of evidence-based treatment for tobacco dependence beyond that provided by certified TTSs. Certification supports an overall higher quality standard of care, ensures that the public receives high-quality treatment, and assist the public and health care organisations in identifying professionals who are competent to deliver intensive evidence-based treatment. Unified certification of TTSs will significantly impact the quality and availability of evidence-based treatments for tobacco dependence. Increasing the quality and availability of evidence-based treatments for tobacco dependence requires a workforce of practitioners with specialist-level proficiency and expertise to provide comprehensive treatment; to provide widespread minimal training of health care practitioners; to guide the integration of tobacco treatment services within dynamic, complex health care systems; and to serve as expert resources for the public. Unified certification of TTSs recognises the competencies required to effectively achieve these components and ensures that ongoing training responds appropriately to changes and needs in the field through iterative review of the competencies and continuing education require- ments. The long-term effects of unified certification of TTSs is likely to impact the current tobacco dependence delivery system and the currently accepted standard of care, yielding improvements in accessibility, the quality of treatment, and the success with which smokers achieve long-term abstinence, all of which will result in improvements in public health and well-being.

\section{Financial Support}

None.

\section{Conflict of Interest}

None.

\section{Ethical Standards}

The authors assert that all procedures contributing to this work comply with the ethical standards of the relevant national and institutional committees on human experimentation and with the Helsinki Declaration of 1975, as revised in 2008.

\section{References}

Anthonisen, N. R., Skeans, M. A., Wise, R. A., Manfreda, J., Kanner, R. E., \& Connett, J. E. (2005). The effects of a smoking cessation intervention on 14.5-year mortality: A randomized clinical trial. Annals of Internal Medicine, 142(4), 233-239.

Applegate, B. W., Sheffer, C. E., Crews, K. M., Payne, T. J., \& Smith, P. O. (2008). A survey of tobacco-related knowledge, attitudes and behaviours of primary care providers in Mississippi. Journal of Evaluation in Clinical Practice, 14(4), 537544. doi: 10.1111/j.1365-2753.2007.00910.x

Backinger, C. L., Thornton-Bullock, A., Miner, C., Orleans, C. T., Siener, K., \& DiClemente, C. C. et al. (2010). Building consumer demand for tobacco-cessation products and services: The national tobacco cessation collaborative's consumer demand roundtable. American Journal of Preventive Medicine, 38(Suppl 3), S307-S311. doi: 10.1016/j.amepre.2009.12.002

Bansal, M. A., Cummings, K. M., Hyland, A., \& Giovino, G. A. (2004). Stop-smoking medications: Who uses them, who misuses them, and who is misinformed about them? Nicotine \& Tobacco Research, 6(Suppl 3), S303-S310.

Borland, R., Partos, T. R., Yong, H. H., Cummings, K. M., \& Hyland, A. (2012). How much unsuccessful quitting activity is going on among adult smokers? Data from the International Tobacco Control Four Country cohort survey. Addiction, 107(3), 673-682. doi: 10.1111/j.1360-0443.2011.03685.x

Centers for Disease Control and Prevention (CDC) (2011). Quitting smoking among adults - United States, 2001-2010. Morbidity and Mortality Weekly Report, 60, 1513-1519.

Chambless, D. L., Baker, M. J., Baucom, D. H., Beutler, L. E., Calhoun, K. S., \& Crits-Christoph, P. et al. (1998). Update on empirically validated therapies, II. The Clinical Psychologist, $51,18$.

Chambless, D. L., \& Hollon, S. D. (1998). Defining empirically supported therapies. Journal of Consulting and Clinical Psychology, 66(1), 7-18. 
Compas, B. E., Haaga, D. A., Keefe, F. J., Leitenberg, H., \& Williams, D. A. (1998). Sampling of empirically supported psychological treatments from health psychology: Smoking, chronic pain, cancer, and bulimia nervosa. Journal of Consulting and Clinical Psychology, 66(1), 89112.

Fiore, M. C., Jaén, C. R., Baker, T. B., Bailey, W. C., Benowitz, N. L., \& Curry, S. J. et al. (2008). Treating tobacco use and dependence: 2008 update. Clinical Practice Guideline. Rockville, MD: Public Health Service.

Houston, T. K., Scarinci, I. C., Person, S. D., \& Greene, P. G. (2005). Patient smoking cessation advice by health care providers: The role of ethnicity, socioeconomic status, and health. American Journal of Public Health, 95(6), 1056-1061. doi: 95/6/1056 [pii] 10.2105/AJPH.2004.039909

Hughes, J. R., Gulliver, S. B., Fenwick, J. W., Valliere, W. A., Cruser, K., \& Pepper, S. et al. (1992). Smoking cessation among self-quitters. Health Psychology, 11(5), 331334

Kasza, K. A., Hyland, A. J., Borland, R., McNeill, A. D., BansalTravers, M., \& Fix, B. V. et al. (2013). Effectiveness of stopsmoking medications: Findings from the international tobacco control (ITC) four country survey. Addiction, 108(1), 193-202. doi: 10.1111/j.1360-0443.2012.04009.x

Levy, D. T., Graham, A. L., Mabry, P. L., Abrams, D. B., \& Orleans, C. T. (2010). Modeling the impact of smokingcessation treatment policies on quit rates. American Journal of Preventive Medicine, 38(Suppl 3), S364-S372. doi: 10.1016/j.amepre.2009.11.016

McDermott, M. S., Beard, E., Brose, L. S., West, R., \& McEwen, A. (2013). Factors associated with differences in quit rates between "specialist" and "community" stop-smoking practitioners in the english stop-smoking services. [Research Support, Non-U.S. Gov't]. Nicotine \& Tobacco Research, 15(7), 1239-1247. doi: 10.1093/ntr/nts262

McMenamin, S. B., Halpin, H. A., \& Bellows, N. M. (2006). Knowledge of Medicaid coverage and effectiveness of smoking treatments. American Journal of Preventive Medicine, 31(5), 369-374. doi: S0749-3797(06)00270-4 [pii] 10.1016/j.amepre.2006.07.015

Minitry of Health (2007). New Zealand Smoking Cessation Guidelines. Wellington: Ministry of Health.

National Institute for Health and Clinical Excellence (NICE) (2013). Smoking Cessation: Supporting People to Stop Smoking. Manchester, UK: National Institute for Health and Clinical Excellence.

Orleans, C. T. (2007). Increasing the demand for and use of effective smoking-cessation treatments reaping the full health benefits of tobacco-control science and policy gains-in our lifetime. American Journal of Preventative Medicine, 33 (Suppl 6), 340-348.

Orleans, C. T., Mabry, P. L., \& Abrams, D. B. (2010). Increasing tobacco cessation in America: A consumer demand perspective. [Introductory]. American Journal of Preventive Medicine, 38 (Suppl 3), S303-S306. doi: 10.1016/j.amepre.2010.01.013

Payne, T. J., Gaughf, N. W., Sutton, M. J., Sheffer, C. E., Elci, O. U., \& Cropsey, K. L. et al. (2014). The impact of brief tobacco treatment training on practice behaviours, selfefficacy and attitudes among healthcare providers. International Journal of Clinical Practice, 68(7), 882-9. doi: 10.1111/ijcp.12386.

Sheffer, C. E., Anders, M., Brackman, S. L., Steinberg, M. B., \& Barone, C. (2012). Tobacco intervention practices of primary care physicians treating lower socioeconomic status patients. [Research Support, N.I.H., Extramural]. American Jouranal of Medicine Science, 343(5), 388-396. doi: 10.1097/MAJ.0b013e3182302749.

Steinberg, M. B., Alvarez, M. S., Delnevo, C. D., Kaufman, I., \& Cantor, J. C. (2006). Disparity of physicians' utilization of tobacco treatment services. [Research Support, N.I.H., Extramural]. American Journal of Health Behavior, 30(4), 375-386. doi: 10.5555/ajhb.2006.30.4.375.

The Canadian Action Network for the Advancement, Dissemination and Adoption of Practice-informed Tobacco Treatment (CAN-ADAPTT) (2011). Canadian Smoking Cessation Clinical Practice Guideline. Toronto, Canada: Action Network for the Advancement, Dissemination and Adoption of Practice-informed Tobacco Treatment, Centre for Addiction and Mental Health. Retrieved from www.can-adaptt.net.

United States Department of Health and Human Services (DHHS) (2014). 2014 Surgeon General's Report: The Health Consequences of Smoking - 50 Years of Progress.

Vidrine, J. I., Shete, S., Cao, Y., Greisinger, A., Harmonson, P., \& Sharp, B. et al. (2013). Ask-Advise-Connect: A new approach to smoking treatment delivery in health care settings. JAMA Internal Medicine, 173(6), 458-464. doi: 10.1001/jamainternmed.2013.3751

Wolff, F., Hughes, J. R., \& Woods, S. (2013). New terminology for the treatment of tobacco dependence: A proposal for debate. Journal of Smoking Cessation, 8(2), 71-75.

World Health Organization (WHO) (2011). World Health Organization Report on the Global Tobacco Epidemic, 2011: Warnings about the dangers of tobacco. World Health Organization. Retrieved from www.who.int.

Zwar, N., Richmond, R., Borland, R., Stillman, S., Cunningham, M., \& Litt, J. (2004). Smoking Cessation Guidelines For Australian General Practice: Practice Handbook. 